\title{
ERRORES GRAMATICALES EN LA EXPRESIÓN ESCRITA DE LOS GRIEGOS APRENDICES DE ELE: ANÁLISIS EN EL NIVEL B1
}

\author{
GRAMMATICAL ERRORS IN THE WRITTEN EXPRESSION OF THE \\ GREEK LEARNERS OF SFL: ANALYSIS AT B1 LEVEL
}

\author{
EIRINI RAMMOU \\ UNED \\ erammou1@alumno.uned.es
}

Fecha de Recepción: 29-09-2018

Fecha de Aceptación: 18-07-2019

\section{RESUMEN}

En este estudio hemos seguido el modelo de Análisis de Errores que constituye una fuente de información importante en el ámbito de la enseñanza-aprendizaje de lenguas extranjeras. El objetivo de este trabajo ha sido investigar los errores gramaticales que cometen con más frecuencia los aprendices griegos de español como lengua extranjera (ELE) en la expresión escrita del nivel B1 del Marco Común Europeo de Referencia para las Lenguas (MCER). Durante el proceso de aprendizaje los alumnos formulan hipótesis mediante la utilización de estrategias y mecanismos psicolingüísticos y por ello nuestro estudio ha profundizado además en la descripción de estas estrategias con el fin de detectar las causas que originan los errores. La clasificación de los errores se ha basado en el criterio lingüístico, descriptivo y etiológico. Los datos obtenidos provienen de producciones escritas reales de los aprendices griegos. Para el análisis hemos aplicado una investigación cuantitativa. En los resultados se proporcionan porcentajes de los errores frecuentes que hemos localizado. Tanto los resultados obtenidos de nuestro análisis e investigación como las conclusiones a

\footnotetext{
1 Doctoranda en la Escuela Internacional de Doctorado de la UNED.
} 
las que hemos llegado resultan relevantes para la prevención de errores gramaticales y la optimización de la enseñanza del español a aprendices griegos.

Palabras Clave: Análisis de Errores; gramática; aprendices griegos; ELE

\section{Abstract}

In this study we have followed the model of Error Analysis that constitutes an important source of information in the field of teaching-learning foreign languages. The aim of this study has been to investigate the grammatical errors most frequently made by Greek learners of Spanish as a foreign language (SFL) in the written expression of the B1 level of the Common European Framework of Reference for Languages (CEFR). During the learning process, students formulate hypotheses through the use of strategies and psycholinguistic mechanisms, which is why our study also goes deeper into the description of these strategies in order to detect the causes that originate the errors. The classification of errors is based on linguistic, descriptive and etiological criteria. The data obtained come from real written productions of the Greek students. For the analysis we have applied a quantitative research. Percentages of the frequent errors we have located are given in the results. The results obtained from our analysis and research and also the conclusions we have reached are relevant for the prevention of grammatical errors and the optimization of teaching Spanish language to Greek learners.

KeYworDS: Error Analysis; grammar; Greek learners; SFL

\section{INTRODUCCIÓN}

El Análisis de Errores (AE) es relevante en el ámbito de la enseñanza de lenguas extranjeras para los profesores, los alumnos y además los investigadores. Proporciona información beneficiosa sobre el desarrollo lingüístico de los alumnos, los mecanismos y las estrategias cognitivas que utilizan durante el proceso de aprendizaje. Los errores muestran los estadios que los alumnos atraviesan en su proceso e intento de aprendizaje de una lengua extranjera, así como el avance que experimentan en sus conocimientos de un nivel a otro y por ello son muy significativos. Corder (1967) ha sido el primero en reconocer y defender la contribución positiva del error en el aprendizaje de lenguas extranjeras. Ha cambiado la visión negativa que se tenía en el pasado de los errores en positiva e imprescindible.

Mediante el AE los profesores se familiarizan con los tipos de desviaciones que los alumnos suelen cometer y es positivo el hecho de que conocen con anticipación las áreas que presentan más dificultades para sus alumnos. Saben cuáles son los aspectos que sus alumnos han asimilado correctamente y pueden poner en práctica y cuáles no. Los profesores pueden prevenir la producción de errores orientando su docencia hacia la resolución 
de los problemas detectados. Esto se consigue también por medio del diseño de actividades adecuadas y de material didáctico adaptado a las necesidades de los alumnos.

Los primeros estudios lingüísticos que abarcaron el concepto del error en el aprendizaje de lenguas extranjeras surgieron a finales de los años 60 con el Análisis Contrastivo (AC). Su objetivo era pronosticar los errores producidos mediante comparación de diferencias y similitudes de la lengua extranjera con la lengua materna de los alumnos. Este enfoque, que recibió muchas críticas, lo sustituyó una nueva corriente de investigación en la Lingüística Aplicada, el AE (Gutiérrez Quintana, 2005).

Corder (1967) sentó las bases del AE. El objetivo principal de esta corriente consiste en identificar la tipología de errores que los aprendices cometen y las causas que provocan las desviaciones, partiendo de las producciones reales de los alumnos (Fernández López, 1997; Alexopoulou, 2011).

Corder (1967), realizó la distinción entre los conceptos de error y falta. Según este autor, el error es una desviación sistemática de la norma de la LE. Los alumnos no pueden autocorregirse porque no han asimilado todo lo que sus profesores les han enseñado (Edge, 1989; Nayernia, 2011). Un ejemplo que se percibe como un error es el siguiente: a) El salón es* \{está $\}$ al lado de la cocina ${ }^{2}$. Por otro lado, la falta es una desviación no sistemática de las reglas de la LE. Los profesores saben que sus alumnos pueden entender sus faltas y por consiguiente pueden autocorregirse (Bustos Gisbert, 1998). La falta sucede "por falta de atención, cansancio, descuido o por algún otro aspecto del desempeño" (Piewo, 2014: 1778). Los siguientes ejemplos se consideran faltas: b) No puedo assistir* $\{$ asistir $\}$ a la clase de español hoy, c) Mi quaderno* $\{$ cuaderno $\}$ es de color azul.

En la presente investigación hemos partido del modelo de AE propuesto por Corder (1967). Las etapas que abarca la metodología del AE según Corder (1971/1991: 72-73) son la identificación de los errores en su contexto o sea de la idiosincrasia producida por los aprendices, la clasificación y descripción de las desviaciones y la explicación de las causas de los errores que guía a las estrategias usadas por los aprendices.

El objetivo del presente estudio es investigar y analizar los errores gramaticales en la expresión escrita del nivel B1 de los aprendices griegos de ELE. Además nuestra investigación tiene como fin detectar las causas que originan los errores mediante el estudio de las estrategias y los mecanismos psicolingüísticos que los alumnos utilizan cuando aprenden una lengua extranjera, siguiendo el criterio etiológico propuesto por Fernández López (1997: 48), basado en ocho categorías a las cuales nos referimos en el apartado de la metodología y en concreto en el método utilizado.

Nuestro estudio es relevante puesto que es una investigación actual, con datos recogidos en el curso 2016-2017. Pretende proporcionar datos e información que contribuirán a la optimización de la enseñanza de ELE en Grecia.

${ }^{2}$ Los ejemplos a), b) y c) han sido extraídos de las producciones de expresión escrita recogidas para la realización de la presente investigación. 
Las hipótesis a las que damos respuesta son:

1) ¿cuáles son los errores gramaticales más frecuentes de los alumnos griegos de ELE en la expresión escrita del nivel B1?

2) ¿cuáles son las causas que originan estos errores?

3) ¿cuáles son las estrategias y mecanismos psicolingüísticos empleados por los aprendices griegos de ELE que provocan los errores?

\section{ESTUDIOS PRECEDENTES}

El precursor del modelo de investigación de la Lingüística Aplicada denominado AE fue Corder en 1967. Desde entonces se han realizado numerosos estudios en todo el mundo que tienen como tema central el AE. Los primeros estudios que se realizaron en torno al AE orientados al ámbito de la enseñanza de ELE fueron las tesis doctorales desarrolladas por Vázquez (1991), Fernández (1991) y Santos Gargallo (1992). Estos trabajos, fueron primordiales para el acercamiento de esta corriente de la Lingüística Aplicada a la enseñanza de ELE.

Hay que señalar que a lo largo de la década de los noventa y sobre todo en la primera década de 2000 se han desarrollado varios trabajos fin de máster en España y tesis doctorales que siguen la metodología del AE en el ámbito de la enseñanza de ELE, dedicados a aprendices de diferentes lenguas maternas (LM). Destacan las investigaciones de aprendices cuya LM es el inglés, el francés o el alemán. No obstante, no faltan las tesis doctorales con aprendices cuya LM es, por ejemplo, el portugués (Torijano Pérez, 2002), el italiano (Sánchez Iglesias, 2003; Gutiérrez Quintana, 2004), el polaco (Fernández Jódar, 2006), el serbio (Alonso Zarza, 2015) o el árabe (Elgamal, 2015), entre otras.

En Grecia, en cuanto a la enseñanza de ELE, pocos son los estudios que han tratado el AE y son destinados a aprendices de ELE con LM el griego. Los estudios que tratan específicamente los errores gramaticales en la expresión escrita de aprendices griegos de ELE no son tantos como en otras lenguas, sin embargo resultan significativos por los datos que cada uno aporta. Hay que señalar que muchos de los estudios realizados en el ámbito griego de ELE se dedican a los errores del léxico.

La mayoría de dichos estudios se realizó desde 2001 hasta 2011 y cabe mencionar que las muestras y datos de algunas de estas investigaciones fueron recogidos durante la década de los 90. Todos los estudios se llevaron a cabo exclusivamente en Atenas. Hay que destacar que en los últimos años (2011-2018) faltan estudios actualizados de AE destinados a aprendices griegos de ELE.

Leontaridi (2001) realiza en su tesis doctoral un estudio basado en el AC de los tiempos del pasado de indicativo del griego moderno y el español. Alexopoulou (2005) en su tesis doctoral estudia los errores morfosintácticos en la interlengua de la expresión escrita de aprendices de español con LM el griego. 
En los artículos de Leontaridi, Peramos Soler y Ruiz Morales (2007 y 2009) se analizan los errores cometidos por alumnos griegos en la prueba de expresión escrita de los exámenes del Diploma de Español como Lengua Extranjera (DELE) de nivel B2 del Instituto Cervantes. En estos estudios, se detectaron errores de varios tipos, a saber, ortográficos, morfosintácticos, léxicos y pragmáticos. En el artículo de 2007 se registraron en general errores de índole morfosintáctica, léxico-semánticos y de pragmática. Sobre los últimos, los problemas se centraron en el uso incorrecto de los verbos ser-estar, habertener, llevar-traer, llevar-tener e ir-venir. En el artículo de 2009 se analizaron errores morfosintácticos y léxicos en los que se observa interferencia de la LM o L2 de los candidatos. Los errores se clasificaron en tres categorías generales: a) errores de omisión, b) errores de redundancia y c) errores de confusión.

Por otra parte los artículos de Palapanidi (2011) y Alexopoulou (2011) tratan el AE en el ámbito léxico-semántico. Del estudio de Palapanidi (2011) se deduce que la idiosincrasia del léxico español y su semántica generan problemas a los aprendices griegos y por ello la mayoría de los errores son intralinguales semánticos. El artículo de Alexopoulou (2011) hace uso del mismo corpus del artículo de Palapanidi (2011) e investiga la transferencia positiva y negativa en la producción de errores léxicos.

Un artículo reciente de López-García y Andreou (2017) analiza los errores en los niveles B1.2 y B2.1 en la expresión escrita de alumnos griego-chipriotas de educación secundaria en Chipre. El español es una asignatura que se imparte en la educación reglada de Chipre, al contrario que en Grecia, donde para aprender español hay que recurrir a institutos privados de enseñanza de ELE. En este estudio se registró un alto porcentaje de errores en el uso de las preposiciones y de las formas verbales, los tiempos, los modos y los verbos ser y estar. La causa de los errores es la utilización de estrategias como la analogía, la neutralización, la interferencia y la influencia de la forma fuerte. Otros errores abarcan el uso incorrecto de los artículos y los adjetivos. Como causa principal se destacó por parte de las autoras la interferencia y los efectos de la adición, la falsa elección, la forma errónea y la colocación falsa.

\section{METODOLOGÍA}

\subsection{Participantes}

En el estudio participaron 87 alumnos griegos que aprendían el español como lengua extranjera en el curso 2016-2017 en 2 institutos privados de aprendizaje de lenguas extranjeras en Tesalónica. El español en Grecia hasta el momento no se enseña en la educación reglada y se enseña solo en institutos privados. Por ello nuestra investigación ha sido llevada a cabo en estos centros educativos. Los institutos han sido seleccionados porque tienen una experiencia de décadas en la enseñanza de la lengua española. En cuanto a los alumnos, 45 provienen del primer instituto y 42 del segundo. 68 de los alumnos son mujeres y 19, hombres. En el estudio participaron alumnos de 18-25 años del nivel B1 según el Marco Común Europeo de Referencia para las Lenguas (MCER) (Instituto Cervantes, 2002). 
En ambos institutos los aprendices durante la investigación cursaban el segundo año de sus estudios de ELE. La LM de todos los alumnos es el griego. Todos los aprendices hablan el inglés como primera lengua extranjera y como segunda o incluso tercera el francés, el alemán o el italiano. Los alumnos tienen 3 horas de clase de español a la semana.

Los manuales utilizados durante la enseñanza eran diferentes en los dos centros educativos y divididos por niveles según el MCER (Instituto Cervantes, 2002) de editoriales españolas especializadas en libros de ELE. Más en concreto, en el primer instituto privado de enseñanza de lenguas extranjeras los libros usados durante el primer año académico fueron Nuevo Español en marcha, niveles A1 y A2, Libro del alumno y Cuaderno de ejercicios de la editorial SGEL (2014). En el segundo año académico continuaron con el libro de la misma serie Nuevo Español en marcha, nivel B1, Libro del alumno y Cuaderno de ejercicios (2014). En el segundo instituto privado durante el primer año académico de aprendizaje de ELE usaron los libros Nuevo Sueña 1, niveles A1 y A2, Libro del alumno y Cuaderno de ejercicios de la editorial Anaya (2015). En el segundo año académico continuaron con el libro de la misma serie Nuevo Sueña 2, nivel B1, Libro del alumno y Cuaderno de ejercicios (2015).

\subsection{Recopilación de datos e instrumentos/materiales}

En el AE se parte de producciones reales de los aprendices, según lo expuesto por Fernández López (1997) y por Alexopoulou (2011). Siguiendo este modelo, el presente estudio se realizó a través de la recopilación de producciones de expresión escrita realizadas por aprendices griegos de ELE en 2 institutos privados de enseñanza de ELE en Tesalónica.

De los 87 alumnos que han participado en la investigación, en total se recogieron 174 producciones de expresión escrita sobre 2 temas diferentes:

a) Escribe una carta a tu mejor amigo/amiga y describe tu casa y tu barrio con detalles (máximo 185 palabras).

b) Escribe una carta a un familiar tuyo para describir cómo pasas tu día y qué haces en tu tiempo libre (máximo 185 palabras).

Esas producciones escritas se escribieron en clase o en casa como tarea.

Se ha designado como tarea en casa porque sirve para comprobar si los alumnos escriben basándose solo en sus conocimientos adquiridos, es decir sin la ayuda de apuntes, diccionarios, los libros de la clase de ELE, gramáticas o de la red.

Los temas elegidos sirven tanto de repaso de aspectos que se enseñan en los niveles A1 y A2, como para abordar los aspectos instruidos en el nivel B1, que para los alumnos constituían en aquella época nuevos conocimientos. Se pretendía averiguar si habían asimilado o no aspectos básicos de los niveles anteriores (A1 y A2) y del nivel B1, si tenían una buena base en estos aspectos o no y si podían ponerlos en práctica en sus producciones escritas de manera correcta. 
Los objetivos lingüísticos de estos ejercicios, basándonos en los descriptores en la expresión escrita del MCER para el nivel B1 (MCER, 2002: 45, 76, 77, 94, 119, 121, 123, $134,223,238)$ son la puesta en práctica mediante la producción escrita de vocabulario relativo a objetos de la casa, adverbios y preposiciones de lugar, tiendas, actividades de ocio, vida cotidiana, narrar, mantener el carácter informal en la carta (p.ej. uso de la segunda persona del singular), estructuras gramaticales que se enseñan en los niveles A1, A2 y B1.

\subsection{Método}

En la presente investigación hemos partido del modelo de AE propuesto por Corder (1967) y Fernández López (1997), este último orientado al ámbito de ELE. Hemos optado por una investigación cuantitativa en la que hemos computado el número de errores gramaticales en las producciones escritas para determinar cuáles son los porcentajes de errores más frecuentes en cada categoría gramatical en el nivel B1. También hacemos una reflexión sobre las causas, las estrategias y mecanismos psicolingüísticos empleados por los alumnos durante el proceso de aprendizaje.

Las etapas que abarca nuestro AE son a) la identificación, b) la descripción, c) la clasificación y d) la explicación, según el modelo de Corder (1967) y Fernández López (1997). A continuación se describe cada una de las 4 etapas de nuestra investigación:

a) La identificación se trata del reconocimiento de la lengua idiosincrásica que usa el alumno en la producción.

b) La descripción de los tipos de errores detectados se lleva a cabo teniendo como base algunas categorías lingüísticas.

c) La clasificación consiste en la descripción más profunda y detallada de las producciones de los alumnos.

d) La explicación constituye el descubrimiento de las causas, las estrategias y los mecanismos que han conducido al alumno a cometer errores.

Para lograr los objetivos de nuestro estudio hemos establecido y aplicado tres criterios. Se trata del criterio lingüístico o gramatical, del criterio descriptivo y del criterio etiológico. Para ello nos hemos basado en la taxonomía de errores según el criterio lingüístico o gramatical propuesto por Fernández López (1997) y Alexopoulou (2005) que nos ayuda en la clasificación de los errores gramaticales localizados en las producciones escritas de los aprendices griegos. Para la descripción de las equivocaciones nos basamos en la tipología de errores presentada por Fernández López (1997), según el criterio descriptivo. Además para la identificación de las causas y estrategias que provocaron los errores cometidos por los aprendices optamos de nuevo por las propuestas de Fernández López (1997), según el criterio etiológico. De esta manera los resultados se presentan de manera organizada, fiable y explicativa.

En lo que se refiere al criterio lingüístico o gramatical de Fernández López (1997) y Alexopoulou (2005) que aplicamos en nuestra investigación, presentamos solo aquellas ca- 
tegorías gramaticales en las que han aparecido errores frecuentes en nuestro corpus. Por ello, nuestra investigación contempla las siguientes categorías gramaticales: la concordancia de género, los verbos ser y estar, las preposiciones, las formas verbales, los tiempos, los modos, los participios, los posesivos, los demostrativos, los pronombres, los indefinidos y los adverbios.

El criterio descriptivo (Fernández López, 1997) consta de las siguientes categorías: 1) omisión, 2) adición, 3) elección falsa, 4) forma errónea y 5) colocación falsa.

En el criterio etiológico los errores se dividen en errores interlinguales causados por interferencia de la LM y en errores intralinguales generados por dos tipos de estrategias, la reducción a un sistema simplificado y la generalización. El criterio etiológico propuesto por Fernández López (1997: 48) se basa en ocho categorías, 1) interferencia, 2) hipergeneralización o generalización, 3) analogía, 4) influencia de la forma fuerte, 5) hipercorrección, 6) neutralización, 7) reestructuración y 8) errores inducidos.

Relevante es el hecho de que en el Plan Curricular del Instituto Cervantes ${ }^{3}$ podemos encontrar las competencias por destrezas y ahí se describen las categorías gramaticales que tienen que adquirir los estudiantes de ELE en el nivel B1.

\section{ANÁLISIS DE LOS RESULTADOS EN LA EXPRESIÓN ESCRITA DEL NIVEL B1}

En el análisis de los resultados obtenidos de nuestra investigación presentamos, describimos y analizamos los más relevantes en cuanto a frecuencia. Presentamos los porcentajes de los errores gramaticales cometidos en la expresión escrita del nivel B1 y ejemplos representativos de esos errores. Hacemos alusión solo a las categorías gramaticales en las que han aparecido errores en nuestro corpus de producciones escritas. Hay que destacar que toda la muestra presenta los mismos errores. También aclaramos las estrategias y los mecanismos psicolingüísticos utilizados por los alumnos que han generado los errores.

En ambos gráficos de los resultados de los dos institutos donde ha sido llevada a cabo la investigación, los porcentajes aparecen en orden descendente. A continuación desglosamos el número de errores teniendo en cuenta un orden decreciente de frecuencia. Entre llaves presentamos en los dos párrafos siguientes el número de las respuestas correctas dadas por los aprendices en cada categoría gramatical.

En el Gráfico 1. Errores gramaticales de los alumnos del primer instituto se puede observar que en el primer instituto (45 alumnos) se han registrado en total 315 errores. El uso incorrecto en lo que respecta a la concordancia de género es el más frecuente, contando con 159 errores de los 315 en total, es decir 50,48\% (159/315) \{10\}; en cuanto a las preposiciones el porcentaje es de 17,78\% (56/315) \{5\}, en los verbos ser y estar es

\footnotetext{
${ }^{3}$ https://cvc.cervantes.es/ensenanza/biblioteca_ele/plan_curricular/niveles/02_gramatica_inventario_b1-b2.htm
} 
de $11,43 \%(36 / 315)\{4\}$ y en las formas verbales $4,76 \%(15 / 315)\{3\}$. En lo que se refiere a los modos el porcentaje es de 3,49\% (11/315) $\{2\}$ y a los tiempos 3,17\% (10/315) $\{2\}$. Estos son los errores gramaticales más relevantes registrados en nuestra investigación y les siguen los errores en el uso de los pronombres $2,22 \%(7 / 315)\{2\}$ y de los posesivos $1,90 \%(6 / 315)\{1\}$. Se ha localizado un número menor de errores en el uso de los demostrativos $1,59 \%(5 / 315)\{1\}$, los participios $1,27 \%$ (4/315) $\{1\}$, los indefinidos con mismo porcentaje que los participios, es decir $1,27 \%$ (4/315) $\{0\}$ y los adverbios $0,63 \%$ $(2 / 315)\{0\}$.

En el Gráfico 2. Errores gramaticales de los alumnos del segundo instituto se puede observar que en el segundo instituto (42 alumnos) se han registrado en total 336 errores. Se han detectado más errores en el uso de la concordancia de género y el porcentaje es de $49,70 \%(167 / 336)\{12\}$, en las preposiciones $17,26 \%(58 / 336)\{7\}$, en los verbos ser y estar $11,30 \%(38 / 336)\{6\}$, en las formas verbales $4,17 \%(14 / 336)\{3\}$, en los modos $3,87 \%(13 / 336)\{2\}$, en los tiempos $3,57 \%(12 / 336)\{2\}$ y en los posesivos $2,38 \%$ (8/336) \{1\}. Estos son los aspectos gramaticales en los que más errores han producido los aprendices griegos de ELE. Continúan errores en el uso de los participios 2,08\% (7/336) $\{2\}$ y los demostrativos $1,79 \%$ (6/336) $\{0\}$. Los alumnos griegos de ELE en el segundo instituto privado han cometido el mismo número de errores en los pronombres y los indefinidos y el porcentaje es de 1,49\% (5/336) \{0\}. Por último aparecen los adverbios con porcentaje $0,89 \%(3 / 336)\{0\}$.

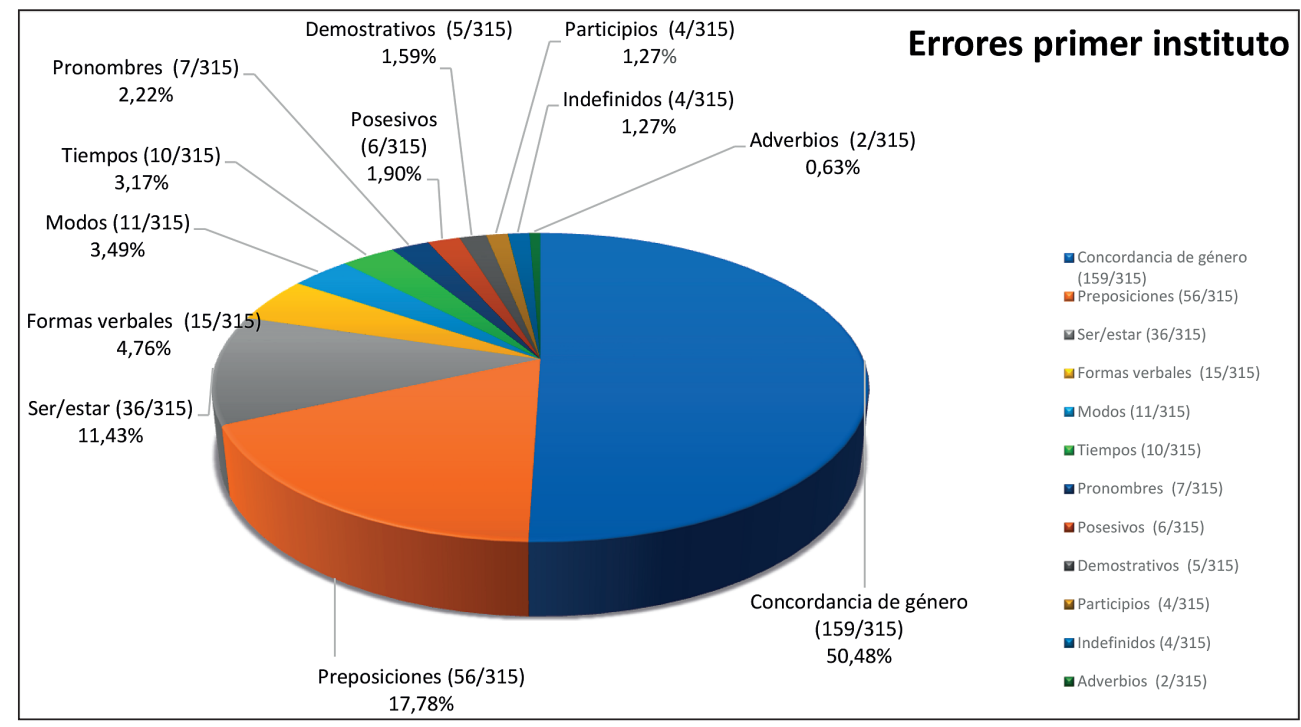

Gráfico 1.

Errores gramaticales de los alumnos del primer instituto 


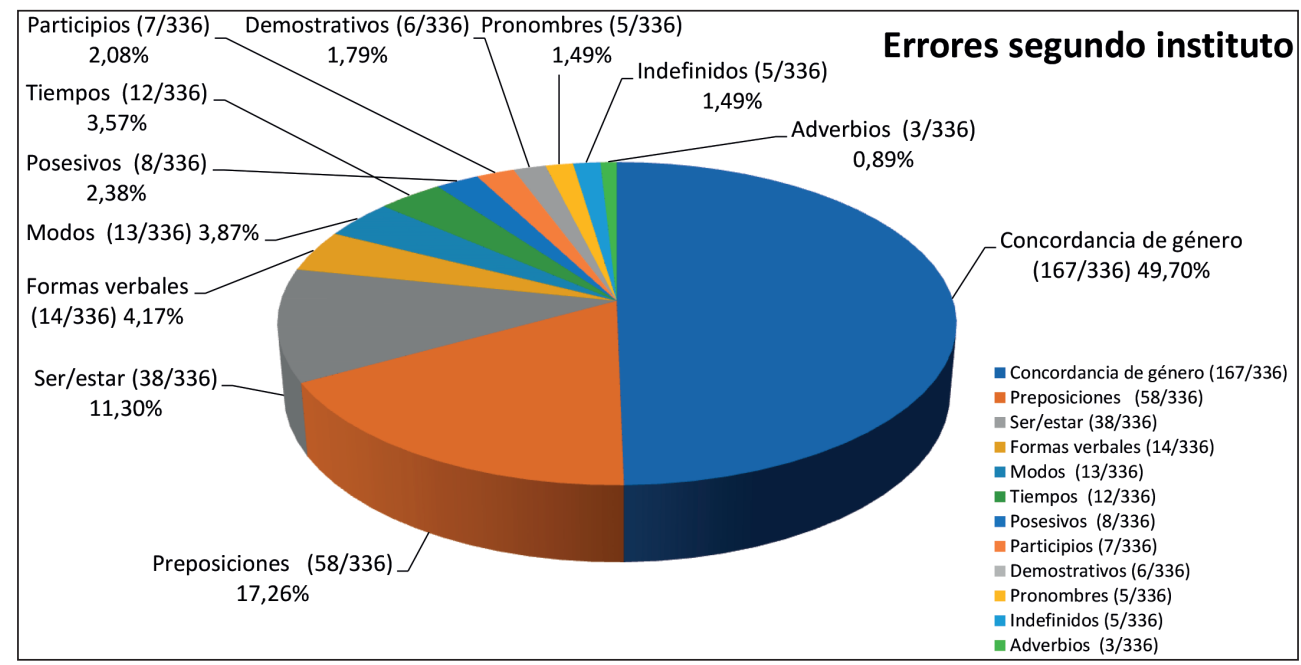

GRÁFICO 2.

Errores gramaticales de los alumnos del segundo instituto

A continuación se presentan los errores más relevantes registrados en las producciones escritas de los aprendices griegos de los dos institutos privados de Tesalónica, clasificados por categorías gramaticales, acompañados de ejemplos representativos y de explicaciones de las causas que provocaron dichas desviaciones. En total los errores cometidos de los 87 alumnos de los dos institutos son 651 .

\subsection{Morfología nominal}

\subsubsection{Concordancia de género}

Se han detectado errores de omisión del artículo determinado (1). En este caso se refleja la neutralización de la función del artículo determinado. Además los alumnos han tenido dificultades en el uso de los géneros del artículo indeterminado (2). El problema aquí reside en el siguiente asunto: en griego las palabras que terminan en -ma pertenecen al género neutro. En español las palabras terminadas en -ma no son de género neutro. Los aprendices no se confunden con el griego, es decir de su LM, sino que se trata de hipergeneralización de las reglas del español, es decir las palabras terminadas en -a - regla general- son de género femenino, así que no se debe a una interferencia del griego.

(1) $\{\mathbf{E l}\} *$ Libro es una buena compañía en mi tiempo libre ${ }^{4}$.

${ }^{4}$ Los ejemplos son extraídos de las producciones escritas recogidas para este estudio. 
(2) El español es una ${ }^{5}\{\text { un }\}^{6}$ idioma que me gusta mucho.

En lo que se refiere a los sustantivos, hemos observado muchos errores en la categoría gramatical o flexiva del género. En este apartado se han cometido errores por hipergeneralización de las reglas aprendidas durante las clases. Fernández Sonsoles (1997: 48) explica el concepto de hipergeneralización de la siguiente manera:

Como mecanismos intralinguales, consideramos las hipergeneralizaciones o generalizaciones de las reglas a casos en que estas no se aplican, regularizando el sistema sin tener en cuenta las restricciones (caso por ejemplo de la regularización de los verbos irregulares), o extendiendo el paradigma a los casos en que el sistema lo posibilita pero la norma no lo ha impuesto ( «carnero» por 'carnicero', «malignosa» por 'maligna').

Por su parte, Alexopoulou (2005: 84-85) presenta la explicación que sigue acerca de la hipergeneralización:

Hipergeneralización es la consecuencia indeseada por aplicar indiscriminadamente el mecanismo de generalización, en el que se extiende, mediante una falsa generalización, el uso de una regla a casos en que esta no se aplica, como producto de regularización de paradigmas a partir de estructuras que se perciben como semejantes.

En los ejemplos que presentamos de nuestro análisis, los alumnos aplican en casi todas las circunstancias la regla general que dicta que los sustantivos terminados en $-\mathrm{o}$ (3) en la mayoría de los casos son de género masculino y los sustantivos terminados en -a (4) y (5) son de género femenino. De esta manera se ve afectada la concordancia de los sustantivos con el artículo que los acompaña. En estos errores pues, el problema radica en la no identificación del género del sustantivo de forma correcta que conduce al uso incorrecto.

(3) Pongo el* $\{$ la $\}$ radio antes de ir a la Universidad.

(4) En mi dormitorio hay una* $\{$ un $\}$ mapa de Grecia.

(5) $\mathbf{L} \mathbf{a}^{*}\{$ el $\}$ problema es grande.

De nuevo se dan casos de desviaciones por la falsa identificación del género del sustantivo que genera problemas en la concordancia. La interferencia con la LM de los aprendices (6) es la que origina dichos errores, puesto que el sustantivo 'el país' en griego es de género femenino.

(6) $\mathbf{L a}^{*}\{\mathrm{El}\}$ país hermosa* $\{$ hermoso $\}$ que quiero visitar es España.

En el ejemplo (7) el error se relaciona de nuevo con la incorrecta concordancia. En este caso se genera analogía.

(7) Los alumnos en mi clase son inteligentos* $\{$ inteligentes $\}$.

\footnotetext{
5 El error aparece en negrita y con un asterisco.

6 Entre llaves se indica la opción correcta.
} 
Teniendo en cuenta las operaciones que abarca el criterio descriptivo propuesto por Alexopoulou (2005: 74-75), se han registrado errores de colocación falsa del adjetivo (8) y (9). Son generados por la interferencia de la LM, porque en griego normalmente se coloca primero el adjetivo y sigue el sustantivo. En español la posposición del adjetivo es más frecuente y ofrece un matiz objetivo al sustantivo al que acompaña. Por otro lado, la anteposición da un matiz subjetivo. La anteposición o posposición del adjetivo depende de razones semánticas. Estos aspectos dificultan a los alumnos griegos de ELE y por ello cometen estos errores.

(8) Inteligente* y guapa* amiga $\{$ Amiga inteligente y guapa $\}$.

(9) Me gusta la casera* comida $\{$ la comida casera $\}$.

\subsection{Verbos}

\subsubsection{Ser / Estar}

Una de las dificultades típicas con la que se enfrentan los aprendices de ELE es la problemática de carácter semántico entre el uso de ser y estar. El problema reside en la falta de riqueza y dominio léxico sobre todo de rasgos semánticos propios del adjetivo atributo que acompaña a estos verbos.

En español se utilizan dos signos lingüísticos distintos, es decir los verbos ser y estar, cada uno de ellos con rasgos semánticos diferentes, mientras que en griego y en otras lenguas, como por ejemplo el serbio (Krstić y Pištignjat, 2013) y el árabe (Alarcón Moreno, 2013), existe solo un lexema. Por ello a los alumnos griegos les resulta muy difícil hacer la distinción semántica entre estas dos formas verbales.

En esta categoría los alumnos siguen estrategias que les conducen a la producción de errores de elección falsa (10), (11), (12), (13) y (14). Se trata de la neutralización de los usos de los verbos ser y estar junto con la interferencia del griego.

(10) Mi compañero es* $\{$ está $\}$ en casa.

(11) Está* $\{$ es $\}$ inteligente.

(12) Soy* $\{$ Estoy $\}$ triste porque no tengo tiempo para salir con los amigos este fin de semana.

(13) Estoy* $\{$ Soy $\}$ de Salónica.

(14) Mis amigos y yo estamos* $\{$ somos $\}$ estudiantes de Química.

\subsection{Preposiciones}

Un error producto de la interferencia de la LM de los alumnos es la omisión de la preposición $a$ (15) delante de un complemento directo o indirecto de persona. En griego no se 
utiliza preposición ante un complemento directo o indirecto de persona en casos como el ejemplo (15) y por ello los aprendices se confunden.

(15) En mi fiesta de cumpleaños siempre llamo* $\{\mathbf{a}\}$ todos mis compañeros.

Además, han aparecido muchos errores de uso de preposición incorrecta porque el verbo rige otro tipo de preposición en ese contexto. Hay ejemplos de errores en los que se usa la preposición en (16) por a con los verbos de movimiento. En otros casos, los aprendices se refieren al espacio usando la preposición $a$ (17) en vez de la preposición en. Dichos errores obedecen a la estrategia de neutralización que utilizan los alumnos porque desconocen las reglas que rigen estas preposiciones.

(16) Voy en* \{a\} casa después de las clases.

(17) La pastelería está $\mathbf{a}^{*}\{$ en $\}$ la calle Dioskouroi, muy cerca de mi casa.

Hemos identificado asimismo muchos errores en el uso de las preposiciones para y por que manifiestan la dificultad que enfrentan los griegos en la práctica. En concreto se observan ocurrencias de elección falsa en las cuales se usa por (18) en lugar de para y viceversa (19) y (20). Las desviaciones son resultado de la estrategia de neutralización seguida por los alumnos. El desconocimiento de las reglas conduce a los alumnos a producciones erróneas.

(18) Estudio español por* \{para $\}$ viajar a España en el futuro.

(19) No solo hablo español. También estudié alemán para* \{por\} 3 años en la escuela.

(20) Siempre paso para* \{ por\} la casa de Fernando cuando termino la clase de español. Su casa está cerca del instituto.

\subsection{Formas verbales}

En algunos casos se ha inducido al error de elección falsa de las formas verbales (21). Las estrategias usadas por los aprendices reflejan la interferencia con la LM. En griego se utiliza el verbo cífal (ser) para expresar la edad como en el ejemplo (21). En el ejemplo presentado se hace uso erróneo del verbo ser en lugar del verbo tener.

(21) Soy* $\{$ tengo $\} 20$ años.

Otros errores localizados se atribuyen a la analogía. Se trata de la confusión entre unas formas verbales parecidas (22) y (23). En estos ejemplos los alumnos confunden la primera persona con la tercera y viceversa en pretérito indefinido.

(22) Fui* \{fue $\}$ una película aburrida.

(23) Fue* $^{*}$ fui $\}$ a casa de mi amiga y cenamos pizza. 
Por otra parte, algunas desviaciones atañen a verbos utilizados de forma errónea en pretérito imperfecto. Estas producciones incorrectas se deben a la sustitución de las desinencias de verbos de la segunda y la tercera conjugación por verbos de la primera conjugación y viceversa (24) y (25). Se sigue por parte de los alumnos la estrategia de generalización.

(24) Nos hablía* \{hablaba $\}$ toda la tarde sobre el examen.

(25) Cuando era niño, en casa comábamos* $\{$ comíamos $\}$ con mis padres pronto.

Otras ocurrencias tienen que ver con la forma errónea de verbos irregulares con alternancia vocálica (por ejemplo de e>ie y de o>ue) del presente de indicativo, como por ejemplo entender (26), encontrar (27), etc. Estos errores se explican por la analogía. Los verbos irregulares del presente de indicativo forman parte de la materia de enseñanza de ELE del nivel A1 (MCER, 2002) y este tipo de errores no son aceptables en el nivel que nos ocupa, puesto que los alumnos ya deberían haber asimilado la formación de los verbos irregulares del presente de indicativo y poder usarlos de manera correcta en el nivel B1.

(26) No entendo* $\{$ entiendo $\}$ todo en la Universidad.

(27) En mi barrio se encontran* \{se encuentran $\}$ muchas tiendas.

Se han generado usos de forma errónea en pretérito indefinido. Los alumnos usan la tercera persona en lugar de la primera (28). Este error tiene su origen a la interlengua y a la analogía. A los alumnos se les enseña primero la conjugación del presente de indicativo y por el tiempo de exposición a este fenómeno gramatical se acostumbran al uso de la desinencia $-\mathrm{o}$ en la primera persona. En etapas posteriores aprenden el pretérito indefinido y les resulta difícil familiarizarse y usar la desinencia -o para la tercera persona. A pesar de que existe el acento gráfico en -ó para el pretérito indefinido no es un aspecto que les ayude a evitar el error finalmente.

(28) Ayer no encontró* $\{$ encontré $\}$ en el supermercado el queso que me gusta.

\subsection{Morfología verbal}

\subsubsection{Tiempos}

En este apartado se dan casos de elección falsa de verbos en pretérito imperfecto (29) en lugar de verbos en pretérito indefinido por neutralización.

(29) Ayer el autobús llegaba* \{llegó $\}$ tarde. 


\subsubsection{Modos}

Los errores en esta categoría gramatical tienen que ver con la elección falsa del modo indicativo en lugar de subjuntivo (30), (31) y se justifican por la hipergeneralización. Los aprendices prefieren la forma menos marcada y más frecuente.

En los demás errores se usa el subjuntivo en vez del indicativo (32) por la estrategia contraria de la hipergeneralización, la denominada hipercorrección.

Se deduce que los aprendices griegos se encuentran en una etapa de su aprendizaje que intentan distinguir entre los usos del indicativo y del subjuntivo y no han asimilado lo que han aprendido durante las clases de ELE, por ello no ponen en práctica de manera correcta sus conocimientos.

(30) Espero que mi compañera aprueba* \{apruebe $\}$ el examen.

(31) ¡Ojalá mañana paso* \{pase \} el examen de literatura en la Universidad!

(32) Cada tarde cuando vuelva* $\{$ vuelvo $\}$ a casa, llamo a mis padres que viven en otra ciudad.

\subsubsection{Participios}

Se presentan errores que afectan a la forma de los participios. Se usa el participio regular en lugar del irregular (33) y (34). Dichas desviaciones se relacionan con la analogía.

(33) He escribido* $\{$ escrito $\}$ el trabajo que espera mi profesor esta semana.

(34) Por la mañana he abrido* $\{$ abierto $\}$ la ventana.

\subsection{Determinativos}

\subsubsection{Posesivos}

Los errores tienen que ver con el uso erróneo del posesivo tu (35) en lugar de su. Son originados de la interferencia con los posesivos de la LM, el griego, donde la segunda persona del singular del posesivo es $\sigma o v$ (se pronuncia su, es decir de manera igual que la tercera persona del singular posesivo en español, su) y la tercera persona del singular del posesivo es $\tau$ ov en masculino y neutro (se pronuncia tu, o sea de la misma manera que la segunda persona del singular posesivo en español, tu). Se trata de interferencia fónica que provoca confusión a los alumnos y les guía al error.

(35) $\mathbf{T} \mathbf{u}^{*}\{$ su $\}$ amiga Ana me cae bien. 
El siguiente tipo de errores procede de la elección falsa del posesivo, algo que es generado por hipercorrección. Se utiliza el pronombre (36) en lugar del adjetivo que debería acompañar el sustantivo.

(36) Es mía* $\{\mathrm{mi}\}$ casa.

\subsubsection{Demostrativos}

Se han recogido casos de forma errónea del demostrativo neutro esto en lugar del masculino este (37) que responden a la estrategia de la analogía. Los aprendices utilizan también el mecanismo de la generalización.

(37) Esto* $^{*}$ Este $\}$ es mi barrio.

\subsection{Pronombres}

La omisión de los pronombres personales (38) y (39) en el uso de los verbos reflexivos se relaciona con la interferencia de la LM de los aprendices. En griego no existen pronombres para los verbos reflexivos, factor que les confunde. Por lo tanto, en griego, los verbos reflexivos disponen de desinencias concretas para cada persona del singular y del plural. Por ejemplo, la desinencia para la primera persona del singular es $-\mu \alpha l$.

(38) Cuando termino las clases en la Universidad quedo* $\{$ me quedo $\}$ en casa para estudiar y preparar la cena.

(39) Levanto* $\{$ Me levanto $\}$ a las 7, lavo* $\{$ me lavo $\}$ y tomo desayuno a las 8.00.

Otros errores tienen que ver con la colocación falsa de los pronombres de objeto/complemento directo o indirecto (40) que es originada por la interferencia de la LM. En griego los pronombres de objeto/complemento directo e indirecto se anteponen al verbo.

(40) A las 16.00 sueles me* encontrar \{encontrarme en la biblioteca de la Universidad estudiando.

Hay que señalar que los pronombres reflexivos se incluyen en la materia de enseñanza de los libros de ELE del nivel A1, según el MCER (2002). En lo que se refiere a los pronombres de objeto/complemento directo, en la mayoría de los libros de ELE aparecen desde el nivel A1 (MCER, 2002). Por otro lado, los pronombres de objeto/complemento indirecto suelen ser instruidos a los alumnos a partir del nivel A2 (MCER, 2002). Los profesores profundizan más en estos dos aspectos gramaticales en el nivel A2 (MCER, 2002). 


\subsection{Indefinidos}

La elección falsa del adverbio тиу (41) en vez del adjetivo indefinido mисho, -os, $-a$, - as y viceversa (42) ha ocasionado errores. Estas desviaciones se provocan por analogía, en concreto por similitud formal y semántica entre el adverbio y el adjetivo, algo que también sucede en la LM de los alumnos. Como no es siempre fácil atribuir una sola causa a cada error, no descartamos en este caso la posibilidad de la interferencia de la LM como factor determinante de producción de este tipo de errores. En griego existe solo una palabra para expresar el mismo significado de mиу у mисho, -os, - $a$, - as. Esta palabra griega mantiene la misma raíz en el tema y tiene diferente terminación dependiendo de su función como adverbio o adjetivo. Por ello los alumnos se guían de su LM, aplican este conocimiento al español y cometen el error.

(41) Muy* \{muchas $\}$ cosas en mi habitación no son útiles.

(42) Mucho* $\{$ muy $\}$ simpático.

\subsection{Adverbios}

Los usos erróneos del adverbio en lugar del adjetivo (43) manifiestan la neutralización de las funciones sintácticas. Estas desviaciones afectan más el tipo bien/buen(o).

(43) Cerca de mi casa hay un bien* \{buen $\}$ supermercado.

Los errores de colocación falsa son cometidos por ignorancia de las restricciones de la regla, especialmente en el pretérito perfecto. Los alumnos usan el adverbio (44) y (45) entre el verbo auxiliar haber y el participio.

(44) Esta tarde con mis compañeros hemos ya* comido $\{$ ya hemos comido $\}$ en el restaurante.

(45) He ya* estudiado \{ya he estudiado $\}$ por* $\{$ para $\}$ el examen.

\section{ERRORES TOTALES}

En el Gráfico 3. Total de los errores gramaticales de los alumnos de ambos institutos vemos que el total de los errores detectados en las producciones escritas de los aprendices de los dos institutos privados es 651. Podemos observar que los resultados en ambos institutos coinciden en cuanto a las categorías gramaticales en las cuales los alumnos cometen más errores frecuentes. Los aprendices de los dos institutos privados cometen más errores en el uso de la concordancia de género con un porcentaje de 50,08\%, 326 errores de los 651 en total (326/651), las preposiciones 17,51\% (114/651) y los verbos ser y estar $11,37 \%$ (74/651). Además se observa dificultad en el uso de las formas verbales $4,45 \%$ 
(29/651), los modos 3,69\% (24/651) y los tiempos 3,38\% (22/651). En menor porcentaje aparecen errores en los posesivos 2,15\% (14/651), los pronombres 1,84\% (12/651), los participios $1,69 \%$ (11/651) y los demostrativos con mismo porcentaje que los participios, o sea 1,69\% (11/651). En el último lugar se encuentran los errores en los indefinidos 1,38\% (9/651) y los adverbios $0,77 \%(5 / 651)$.

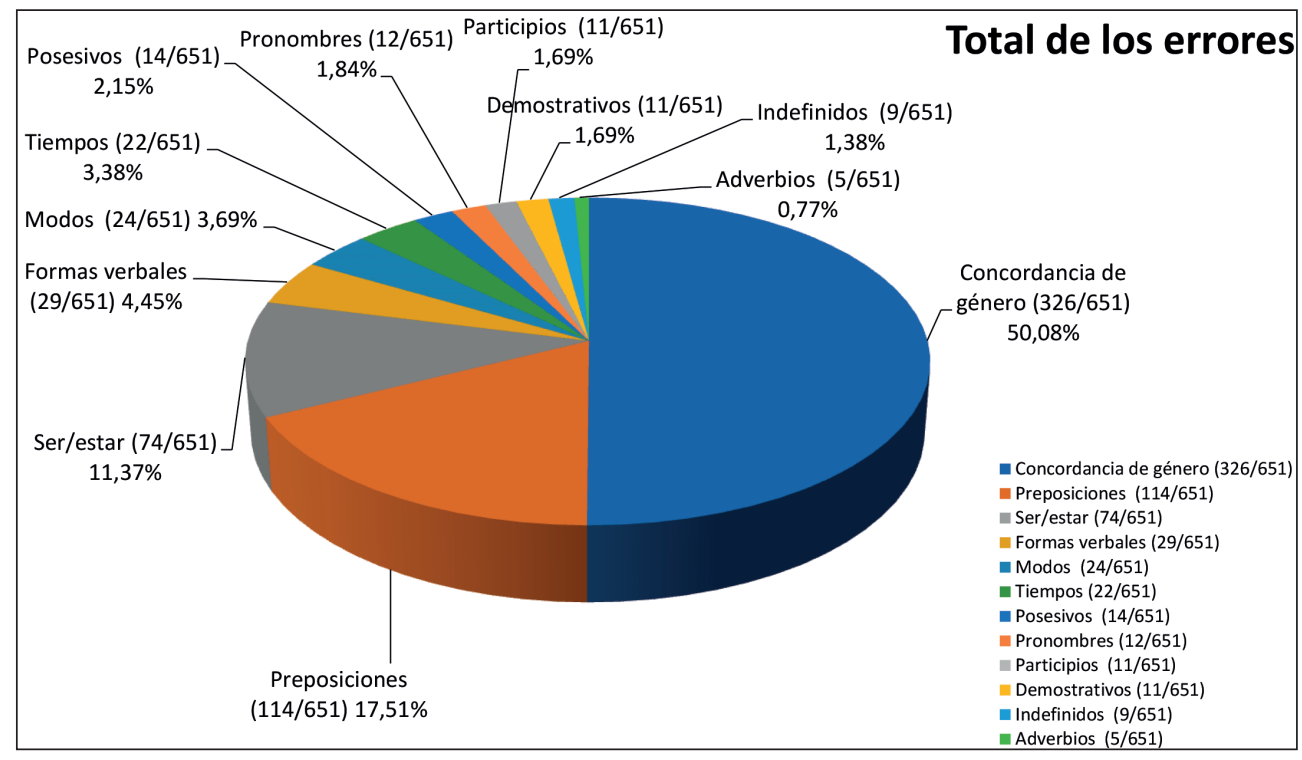

GRÁFICO 3.

Total de los errores gramaticales de los alumnos de ambos institutos

\section{CONCLUSIONES}

El objetivo de este estudio ha sido investigar los errores gramaticales frecuentes de los alumnos griegos de ELE en la expresión escrita del nivel B1, así como las causas y estrategias que originan estos errores.

Del análisis realizado en las producciones escritas se concluye que los alumnos han cometido errores que se engloban no solo en el nivel B1, sino también en los niveles A1 y A2. Esto demuestra que dichos estudiantes no habían asimilado de manera satisfactoria ciertos aspectos gramaticales que se aprenden en los niveles previos al B1.

Los resultados de esta investigación han demostrado que los errores gramaticales más frecuentes que cometen los aprendices griegos en el nivel B1 están relacionados con la concordancia de género, las preposiciones, los verbos ser y estar, las formas verbales, 
los modos, los tiempos, los posesivos, los pronombres, los participios, los demostrativos, los indefinidos y los adverbios. Detalladamente, los aspectos gramaticales en los que los aprendices griegos cometen más errores frecuentes son la concordancia de género, las preposiciones y los verbos ser y estar. Estos errores se deben a ciertas estrategias utilizadas por los alumnos como la analogía, la hipergeneralización, la hipercorrección, la neutralización y la interferencia del español con la LM de los aprendices, el griego. Además causas como la elección falsa, la omisión, la forma errónea y la colocación falsa provocan varias desviaciones.

Los resultados de esta investigación pueden resultar útiles para la optimización de la enseñanza de ELE a alumnos griegos. Las conclusiones de este estudio aportan datos relevantes para los profesores puesto que les ofrecen información que puede contribuir a la prevención de errores gramaticales, a la superación y a la disminución de los mismos mediante el diseño de actividades didácticas adecuadas. También pueden ayudar a los diseñadores de los programas de estudio y los autores de materiales didácticos a prestar más atención a los aspectos gramaticales que les resultan más difíciles a los alumnos, con el fin la prevención de los errores.

El reducido número de estudios sobre el análisis de errores gramaticales de los alumnos griegos de ELE y especialmente sobre el nivel B1 crea la necesidad de efectuar más estudios especializados sobre este tema con el fin de mejorar las metodologías de enseñanza y los programas educativos de ELE. Asimismo este estudio de análisis de errores gramaticales puede servir de ayuda para todos los aprendices de ELE, cualquiera que sea su LM. Y, por último, esperamos que pueda servir de base e inspiración para futuras investigaciones.

\section{REFERENCIAS BIBLIOGRÁFICAS}

Alarcón Moreno, Mauricia (2013). Análisis de errores en la interlengua de aprendientes sirios de español como lengua extranjera, tesis doctoral, Madrid, Universidad Autónoma de Madrid.

Alexopoulou, Angélica (2005). Contribución al estudio de los errores morfosintácticos en la interlengua escrita de aprendientes de español de origen griego, tesis doctoral, Atenas, Universidad Nacional y Kapodistríaca de Atenas, recuperado de http://thesis.ekt.gr/thesisBookReader/ id/22296\#page/1/mode/2up, consultado el 20 de octubre de 2017.

Alexopoulou, Angélica (2011). "El papel de la transferencia en los errores léxicos", en Revista Nebrija de Lingüística Aplicada, 9: pp. 27-36, recuperado de http://www.nebrija.com/revista-linguistica/numero9/numero9/pdf/Alexopoulou1.pdf, consultado el 26 de octubre de 2017.

Alonso ZarZa, María Ángeles (2015). Análisis de errores en oraciones condicionales y concesivas en aprendientes de ELE serbohablantes, Madrid, Universidad Autónoma de Madrid.

Álvarez Martínez, Ma Ángeles ; Blanco Canales, Ana ; Gómez Sacristán, Mª Luisa ; Pérez De La Cruz, Nuria (2015). Nuevo Sueña 1, niveles A1-A2, Libro del alumno, Madrid, Anaya.

Álvarez Martínez, Ma Ángeles ; Blanco Canales, Ana ; Gómez Sacristán, Ma Luisa ; Pérez De La Cruz, Nuria (2015). Nuevo Sueña 1, niveles A1-A2, Cuaderno de ejercicios, Madrid, Anaya.

Bustos Gisbert, José Manuel (1998). "Análisis de errores: problemas de categorización”, Dicenda, Cuadernos de Filología Hispánica, 16: pp. 11-40, recuperado de http://revistas. ucm.es/index.php/ 
DICE/article/view/DICE9898110011A/12724, consultado el 11 de noviembre de 2017.

Cabrerizo Ruiz, Ma Aranzazu ; Gómez Sacristán, Ma Luisa ; Ruiz Martínez, Ana M. a (2015). Nuevo Sueña 2, nivel B1, Libro del alumno, Madrid, Anaya.

Cabrerizo Ruiz, Ma Aranzazu ; Gómez Sacristán, Mª Luisa ; Ruiz Martínez, Ana M. ${ }^{a}$ (2015). Nuevo Sueña 2, nivel B1, Cuaderno de ejercicios, Madrid, Anaya.

Castro, Francisca ; Díaz, Pilar ; Rodero, Ignacio ; Sardinero, Carmen (2014). Nuevo Español en marcha Básico, niveles A1-A2, Libro del alumno, Madrid, SGEL.

Castro, Francisca ; Díaz, Pilar ; Rodero, Ignacio ; Sardinero, Carmen (2014). Nuevo Español en marcha Básico, niveles A1-A2, Cuaderno de ejercicios, Madrid, SGEL.

Castro, Francisca; Benítez, Ma Teresa ; Rodero, Ignacio ; Sardinero, Carmen (2014). Nuevo Español en marcha 3, nivel B1, Cuaderno de ejercicios, Madrid, SGEL.

Castro, Francisca ; Rodero, Ignacio ; Sardinero, Carmen (2014). Nuevo Español en marcha 3 , nivel B1, Libro del alumno, Madrid, SGEL.

CORDER, Pit StePhen (1967). “The significance of learner's errors”, en IRAL, 5: pp. 161-170.

CORDER, Pit StePhen (1981). Error analysis and interlanguage, Oxford, Oxford University Press.

EdGE, Julian (1989). Mistakes and correction, Harlow, Longman.

Elgamal, Hazem Abdellah (2015). Análisis de los errores morfosintácticos en la interlengua de los estudiantes egipcios de español como lengua extranjera, tesis doctoral, Sevilla, Universidad Pablo de Olavide.

FERNÁNDEZ JódAR, RAúl (2006). Análisis de errores léxicos, morfosintácticos y gráficos en la lengua escrita de los aprendices polacos de español, tesis doctoral, Poznan, Polonia, Universidad Adam Mickiewicz.

FERnÁNDEZ LóPEZ, Sonsoles (1991). Análisis de errores e Interlengua en el aprendizaje del español como lengua extranjera, tesis doctoral, Madrid, Universidad Complutense.

FERNÁNDEZ LóPEZ, SONSOLEs (1997). Interlengua y análisis de errores en el aprendizaje del español como lengua extranjera, Madrid, Edelsa.

Gutiérrez Quintana, Esther (2004). Análisis de errores gramaticales de italianos estudiantes de español, Alcalá de Henares, Universidad de Alcalá.

Gutiérrez Quintana, Esther (2005). Análisis de la interlengua de italianos aprendices de español, Estudios de lingüística, 19, pp. 223-242.

Instituto CeRvantes (2002). Marco común europeo de referencia para las lenguas: aprendizaje, enseñanza, evaluación, en Centro Virtual Cervantes, pp. 1-263, Madrid, Ministerio de Educación, Cultura y Deporte, recuperado el 5 de noviembre de 2017 de http://cvc.cervantes.es/ensenanza/ biblioteca_ele/marco/cvc_mer.pdf, consultado el 15 de octubre de 2017.

Krstić, Katarina Y Pištignjat, Tijana (2013). Análisis de errores en el aprendizaje del español por alumnos que tienen como lengua materna el serbio, Colindancias: Revista de la Red Regional de Hispanistas de Hungría, Rumanía y Serbia, 4: pp. 393-404.

LeONTARIDI, Eleni (2001). Los tiempos del pasado del indicativo en español y en griego moderno, tesis doctoral, Salamanca, Universidad de Salamanca, recuperado de http://phdtheses. ekt.gr/eadd/ handle/10442/22649, consultado el 29 de octubre de 2017.

Leontaridi, Eleni, Peramos Soler, Natividad y Ruiz Morales, Marina (2007). "Análisis y clasificación de errores en la producción de textos escritos de candidatos griegos en los exámenes DELE", Actas del XVIII Congreso Internacional de la Asociación para la Enseñanza del Español como lengua Extranjera (ASELE), Alicante, Universidad de Alicante, pp. 357-364.

Leontaridi, Eleni, Peramos Soler, Natividad y Ruiz Morales, Marina (2009). "Errores en la interlengua escrita de estudiantes grecófonos de español como lengua extranjera", en Zona próxima 11, recuperado de http://rcientificas.uninorte.edu.co/index.php/zona/article/ view/1049/654, consultado el 5 de noviembre de 2017. 
López-García, María Pilar y Andreou, Elefthería (2017). "La Expresión Escrita en Alumnos Griego-Chipriotas de Español: Análisis de Errores en los Niveles B1.2 y B2.1", Revista Porta Linguarum, Monográfico II, 231-244.

NAYERnia, AKRAm (2011). "Writing errors, what they can tell a teacher?", en MJAL, 3, 2: pp. 200-217.

PALAPANIDI, KIRIAKí (2011). "Los errores léxicos intralinguales semánticos en la producción escrita de los aprendientes griegos de español", en Revista Nebrija de Lingüística Aplicada: 9, 5: pp. 2-21, recuperado de http://www.nebrija.com/revista-linguistica/numero9/numero9/pdf/ Palapanidi. pdf, consultado el 12 de noviembre de 2017.

Piewo, SoKeng. (2014). Grammatical errors of bilingual 1 francophone learners of english in the University of Yaounde I. Theory and Practice in Language Studies, 4 (9), pp. 1778-1785.

SÁnchez Iglesias, JoRge (2003). Errores, corrección y fosilización en la didáctica de lenguas afines: Análisis de errores en la expresión escrita de estudiantes italianos de ELE, tesis doctoral, Salamanca, Universidad de Salamanca.

Santos Gargallo, Isabel (1992). La enseñanza de segundas lenguas. Análisis de errores en la expresión escrita de estudiantes de español cuya lengua nativa es el serbo-croata, tesis doctoral, Madrid, Universidad Complutense.

Torijano Pérez, José Agustín (2002). Análisis teórico práctico de los errores gramaticales en el aprendizaje del español, L2: Expresión escrita, tesis doctoral, Salamanca, Universidad de Salamanca.

VÁzQuez, Graciela (1991). Análisis de errores y aprendizaje de español lengua extranjera, (Análisis, explicación y terapia de errores transitorios y fosilizables en el proceso de aprendizaje de español como lengua extranjera en cursos universitarios para hablantes nativos de alemán), Berlin, Peter Lang. 
